\title{
Reconstructive surgery of true aneurysm of the radial artery: A case report
}

\author{
Sevinc Bayer Erdogan, Serdar Akansel, Nehir Tandogar Selcuk, Serap Aykut Aka \\ Department of Cardiovascular Surgery, Siyami Ersek Hospital, Istanbul, Turkey
}

\begin{abstract}
True radial artery aneurysms are uncommon pathologies and have an organic cause, unlike trauma-induced false aneurysms. A 52-year-old man presented with a pulsatile mass at the anatomical snuff box area of his left hand. The aneurysm was repaired with reconstructive procedure. Although many posttraumatic and iatrogenic cases of false aneurysm of the radial artery have been reported; there are a few reported cases of a true idiopathic aneurysm. A case of reconstructive surgery for true idiopathic radial artery aneurysm is reported in this paper.
\end{abstract}

Keywords: Radial artery aneurysm; reconstructive surgery; true aneurysm.

U pper extremity arterial aneurysms are uncommon pathologies with true radial artery aneurysm being the rarest among this group [1]. A true aneurysm is caused by degenerative, congenital, metabolic disorders or can be part of a systemic disease [2]. It is caused by structural deterioration of the vessel wall. All three layers of the arterial wall can be seen under histologic examination.

A delay in surgical intervention is not recommended in these cases because of the risk for thromboembolism or rupture [3]. A case of reconstructive surgery for true idiopathic radial artery aneurysm is presented in this paper.

\section{CASE REPORT}

A 52-year-old, left-hand-dominant man presented to our outpatient clinic with a pulsatile and painful mass at the anatomical snuff box area of his left hand. The mass had been present for 1 year and had gradually enlarged. The patient denied history of trauma; there was no family history of aneurysms.
On physical examination, a spherical mass of size $1.5 \times 2 \mathrm{~cm}$ was found at the anatomical snuffbox area of the patient's left hand without any scars hinting at previous trauma (Fig. 1). There were no signs of other arterial aneurysms on examination. Computed tomography (CT) angiography showed a saccular aneurysm with a size of $14 \mathrm{~mm}$ at the distal part of the radial artery localized on the dorsal aspect of the first carpometacarpal joint (Fig. 2). Because of abnormal preoperative Allen's test, revascularization was planned with primary or graft interposition based on anatomy of the radial artery.

Under local anesthesia, the skin was incised over the aneurysm site. Proximal and distal arterial segments of the aneurysm were encircled with vessel loops. The aneurysm sac was dissected from the surrounding tissue (Fig. 3a). After heparinization and arterial clamping, the aneurysm sac was resected. Then, the reconstruction was performed with primary end-to-end anastomosis following distal and proximal liberalization from the surround-

Received: March 17, 2017 Accepted: May 23, 2017 Online: January 16, 2018

Correspondence: Dr. Serdar AKANSEL. Dr. Siyami Ersek Hastanesi, Kalp ve Damar Cerrahisi Bolumu, Istanbul, Turkey. 


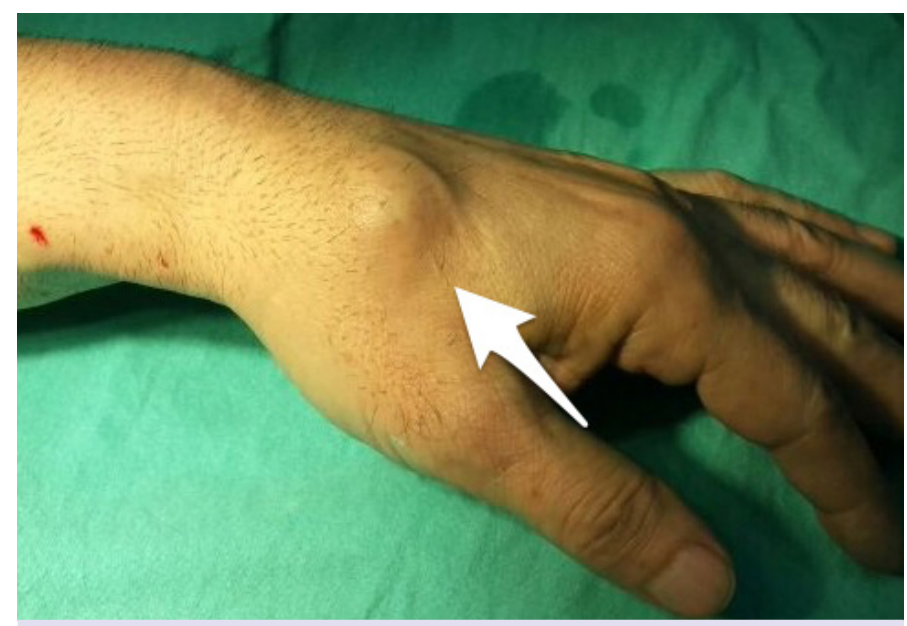

FIGURE 1. A mass at the snuff box area of the left hand.

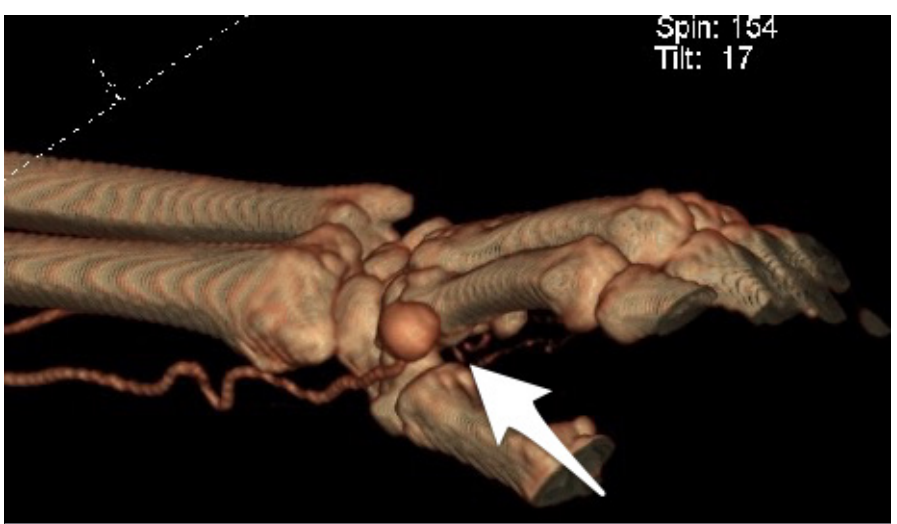

FIGURE2. Reconstructed three-dimensional image of the left hand shows a saccular aneurysm on the radial artery.

ing tissues to allow for a tension-free anastomosis. Blood flow was successfully re-established to the distal segment (Fig. 3b).

The histology of the resected aneurysm showed thrombus formation, fibromyxoid degeneration of the vessel wall, atrophy of the medial layer, and destruction of the elastic lamina. The patient was uneventfully discharged from the hospital on postoperative day 3. No lesion was detected in CT angiography performed 3 months after surgical treatment.

\section{DISCUSSION}

The upper extremity arterial aneurysms are extremely rare entities, and radial artery aneurysm is the rarest form of upper extremity aneurysms [1]. Vessels with a small lumen, such as the radial artery, have a low possibility of aneurysm formation according to Laplace's law
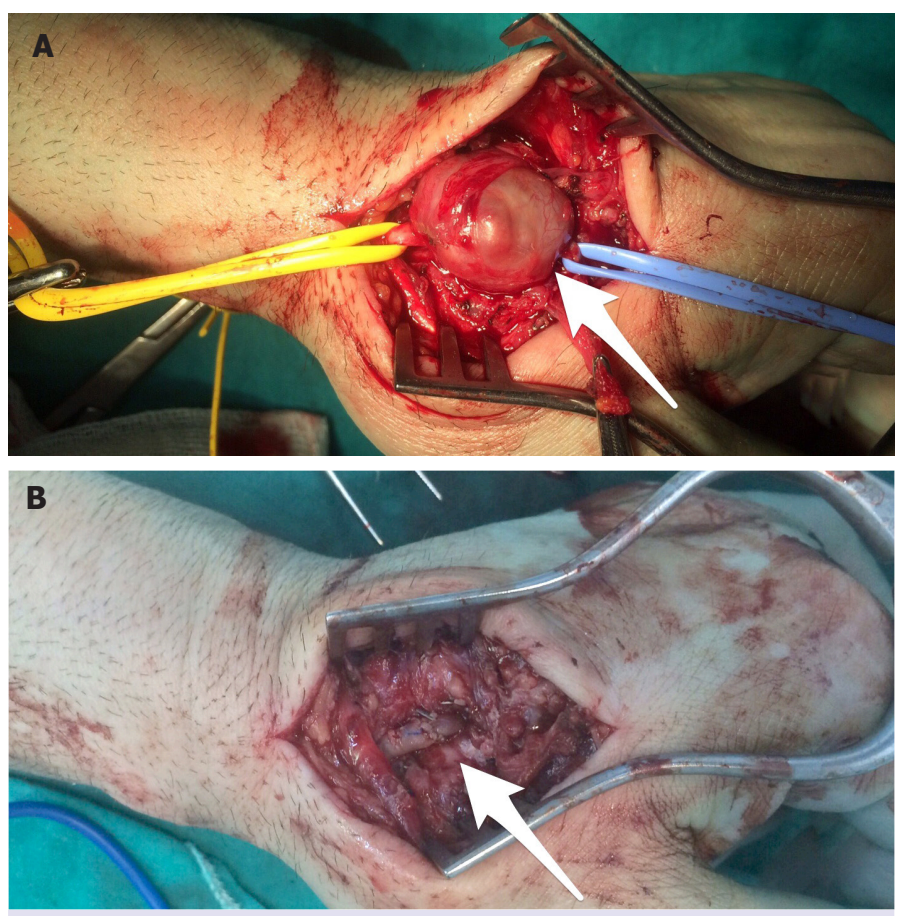

FIGURE 3. Exploration of the aneurysm controlled with vessel loops proximally and distally (a), reconstruction of the radial artery with a primary end-to-end anastomosis (b).

as they require higher pressures to enlarge. This is the reason for uncommon occurrence of aneurysms in small vessels, such as the radial, ulnar, and digital arteries [4].

The diagnosis is initially clinical, but is afterwards confirmed using various imaging modalities such as duplex ultrasonography and CT angiography. Duplex ultrasonography is traditionally used first to help diagnosis as it is inexpensive and easy to access. CT angiography is ideal for whole-body scans to exclude other aneurysms. Magnetic resonance angiography or conventional angiography has also been reported to be used in diagnosis.

Igari et al. [5] reported five patients with upper extremity aneurysms including two brachial, two ulnar, and one radial aneurysm treated with surgical resection. They performed reconstruction for two brachial aneurysms and ligation for the others. The surgical treatment options in the radial and ulnar artery aneurysms depend on whether they dominantly supply blood to the hand. Many options for evaluation of dominance are available with variable sensitivity and specificity: Allen's test, modified Allen's test, digital plethysmography, digital Doppler waveforms and pressures, and duplex ultrasonography [6]. The surgeon can perform a simple resection and ligation on the basis of physical findings and radiographic results. In contrast, a reconstructive surgery can be performed with 
a primary end-to-end anastomosis if there is no tension, or with graft interposition in case the defect is lengthy.

A less invasive endovascular intervention using stent grafts is an alternative treatment option for radial artery aneurysms is, but not many cases of radial artery aneurysms treated with stent grafts have been reported [7]; furthermore, it remains a controversial option.

The present patient had degenerative true radial artery aneurysm. He denied history of trauma and had no family history of aneurysm. First, we performed duplex ultrasonography to confirm the final diagnosis and then CT angiography to exclude other aneurysms. The patient was treated with a reconstructive procedure. Primary end-to-end anastomosis was performed without tension; histologic examination showed fibromyxoid degeneration of the vessel wall.

Although there are alternative treatment options such as simple resection and ligation or endovascular intervention for radial artery aneurysms, this paper favors that reconstructive surgery of the radial artery aneurysms can be safely performed and should be the preferred option, especially in young patients.
Conflict of Interest: No conflict of interest was declared by the authors.

Financial Disclosure: The authors declared that this study has received no financial support.

Authorship contributions: Concept - S.A., S.B.E.; Design - S.A.; Supervision - S.A.A.; Materials - S.A.; Data collection \&/or processing - S.A., N.T.; Analysis and/or interpretation - S.A.; Writing - S.A., S.B.E.; Critical review - S.A., S.A.A.

\section{REFERENCES}

1. Ho PK, Weiland AJ, McClinton MA, Wilgis EF. Aneurysms of the upper extremity. J Hand Surg Am 1987;12:39-46. [CrossRef]

2. Rasouli MR, Moini M, Khaji A. Civilian traumatic vascular injuries of the upper extremity:report of the Iranian national trauma project. Ann Thorac Cardiovasc Surg 2009;15:389-93.

3. Tsutsumi K, Saito H, Ohkura M. Traumatic pseudoaneurysm of the subclavian artery following anterior dislocation of the shoulder: a report of a surgical case. Ann Thorac Cardiovasc Surg 2006;12:74-6.

4. Turner S, Howard CB, Dallimore NS. A case report of a true aneurysm of a digital artery. J Hand Surg Br 1984;9:205-6. [CrossRef]

5. Igari K, Kudo T, Toyofuku T, Jibiki M, Inoue Y. Surgical treatment of aneurysms in the upper limbs. Ann Vasc Dis 2013;6:637-41. [CrossRef]

6. Habib J, Baetz L, Satiani B. Assessment of collateral circulation to the hand prior to radial artery harvest. Vasc Med 2012;17:352-61. [CrossRef]

7. Carrafiello G, Laganà D, Mangini M, Fontana F, Recaldini C, Piacentino F, et al. Percutaneous treatment of traumatic upper-extremity arterial injuries: a single-center experience. J Vasc Interv Radiol 2011;22:34-9. 